\title{
Corona Virus Symptoms and Interpretation through Artificial Intelligence: A case study of India
}

\author{
Richa Pandey, BIT Mesra \& India, richapandey@bitmesra.ac.in \\ Ankita Banerjee, BIT Mesra \& India, ankitabanerjee4u@gmail.com \\ Tathagata Mukherjee, BIT Mesra \& India, tatha95@gmail.com
}

\begin{abstract}
Coronavirus or COVID-19 is a new type of disease which is spreading rapidly by human contact and has been declared as a pandemic by the World Health Organization (WHO). In this paper several parameters like age, cough, cold, travel history, contact with an infected family member, breathing trouble and fever are taken into consideration for predicting the spread of this disease. A survey of one hundred people from 8 different states of India namely Maharashtra, Gujrat, Tamil Nadu, Kerala, West Bengal, Assam, Jammu \& Kashmir and Himachal Pradesh have been taken into consideration, and based on those values the spread of Coronavirus in different states of India has been calculated using MATLAB fuzzy logic. Prediction of the spread of novel Coronavirus can be predicted using fuzzy logic and based on this prediction further preventive steps can be taken by the higher authorities. Conclusive result indicate that North Eastern states are less likely to be diagnosed with Coronavirus than the Western states of the country.
\end{abstract}

Keywords — artificial intelligence, coronavirus, diagnosis, fuzzy logic, pandemic, World Health Organization (WHO).

\section{INTRODUCTION}

Epidemics are a form of natural disaster which takes place without any warning. In the present scenario novel Coronavirus is spreading rapidly through human contact [1]. With the advancement in artificial intelligence and machine learning a fast-growing epidemic can be diagnosed and based on that diagnosis preventive measures can be adopted to suppress the spread [2]. Coronavirus (covid19) is an infectious disease which has recently originated in Wuhan the capital of Hubei province in China. Its rapidly spreading nature through contact had made it a tremor among the population of the world. The world health organization (WHO) has declared it as a pandemic, as no vaccination has been invented as of now [3]. Originating in Wuhan of China, the novel Coronavirus spread rapidly across the globe [4]. The Coronavirus can be seen passing from people to people through contact as it is transmitted by touch or exchange of fluid so the most effective prevention measures adopted by counties to suppress the mass spreading of the novel coronavirus in the absence of vaccination is to practice social distancing [It has been declared by WHO ,(5)]. Countries have adopted rigorous measures to suppress the massive outbreak of this devastating virus. The concealment of social contact in work environments, schools, offices and other open circles is the objective of such measures. Since social contacts have a solid assortative structure in age, the viability of these measures is subject to both the age structure of the population and the recurrence of contacts between age bunches over the population [6].
Quantitative evaluations of the effect of these measures in decreasing death rates, morbidity, infection rates etc. and therefore can be a critical guide in public health planning.

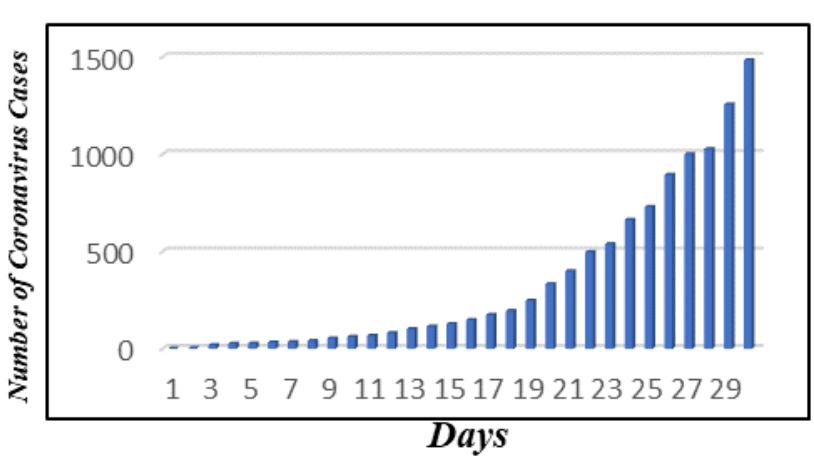

(a)

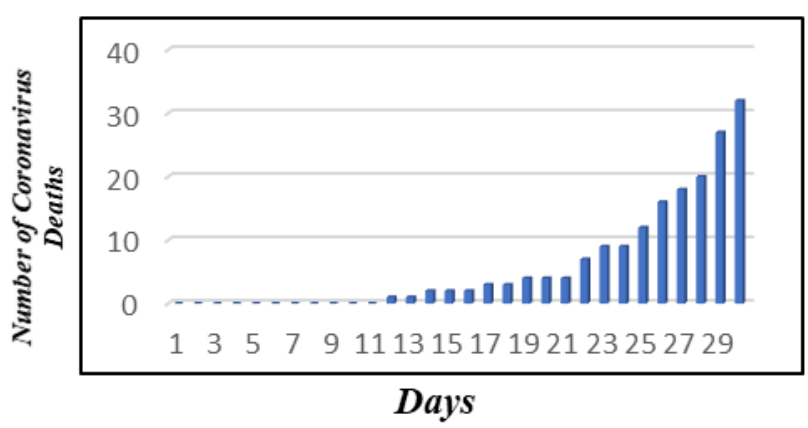

(b)

Fig 1: (a) \& (b) represents total number of Coronavirus cases and total number of Deaths caused by Coronavirus in the month of March The above graphs [7] show the number of people affected by Coronavirus in India in a span of one month from $1^{\text {st }}$ of 
March till $31^{\text {st }}$ of March. Figure "a" represents the number of Coronavirus cases recorded in India and figure "b" represents the total number of deaths due to Coronavirus. Both the graphs clearly predict the exponential rise in Coronavirus cases and the increasing number of people getting infected by this pandemic disease. India witnessed their first death of a civilian from Karnataka by Coronavirus on $12^{\text {th }}$ March 2020 [8]. This spreading of the disease can be possible by mass lockdown and avoid close proximity of infected people as much as possible [9]. There is no method of calculating and predicting the exact next patient of this disease [10], however the fuzzy logic approach can be used to have a rough idea and predict the infection possibility of a person by this disease.

Fuzzy logic is a computational approach to calculate the degree of truth or chance of truth rather than determining the actual answer in Boolean form (True or False \& 0 or 1 form) on which logic modern computer is based [11]. Fuzzy logic determines the possibility of a particular action based on various other actions which are directly or indirectly or partially related to the input [12].

In this paper we present a case study of 8 states in India and respective possibility of a person getting infected in each of these eight states based on few important factors based on which the novel coronavirus infection can be determined. This way a precautionary step can be taken depending on the risk level as calculated by MATLAB Fuzzy logic preventing further outbreak of this epidemic among the population of the country can be stopped.

\section{METHODOLOGY AND CALCULATION}

There are several factors affecting the cause and spread of Coronavirus among people. China being the birth place of this infectious disease, it got spread to other parts of the world by passing from one person to another [13]. Coronavirus interpretation is predicted using fuzzy logic in MATLAB. The symptoms of the disease are considered as the input of the fuzzy logic and the percentage chance of getting infected by this virus. One of the main factors of getting into critical condition due to this virus is the age of a person. According to World Health Organization (WHO), chances of a patient getting into critical condition is if the patient is and elderly person and also have a history of foreign travel background [14]. Few of the highest impacted symptoms of Coronavirus are considered as the basic inputs based on which prediction of infection is computed. In our paper the five basic symptoms and high-risk factor for getting infected from this disease is considered. They are: Cough, Fever, Breathing Issues, International Travel Background and Age [15]. These are the primary factors based on which the percentage of getting infected is calculated using fuzzy logic.

Certain parameters are set to the input variables so that a better prediction can be made. Setting certain member function to each of these inputs will help in obtaining a more accurate prediction of the risk of being infected. Triangular member function [16] of three members have been used in all cases to have uniformity in computing. The table shows the input factors which have been taken into consideration and their respective member functions [17].

TABLE 1: RANGE OF MEMBER FUNCTIONS

\begin{tabular}{|c|c|c|c|}
\hline Parameters & Low & Medium & High \\
\hline Cough & $0-1$ & $0.5-2.5$ & $2-3$ \\
\hline Fever & $0-1$ & $0.5-2.5$ & $2-3$ \\
\hline Breathing & $0-1$ & $0.5-2.5$ & $2-3$ \\
\hline International Travel & $6-10$ & $3-7$ & $0-4$ \\
\hline Age & $0-30$ & $25-60$ & $45-100$ \\
\hline Infected Family & 0 & - & 1 \\
\hline
\end{tabular}

Parameters Low Medium High

Cough 0-1 0.5-2.5 2-3

Fever $\quad 0-1 \quad 0.5-2.52-3$

Breathing 0-1 0.5-2.5 2-3

International Travel6-10 3-7 0-4

Age 0-30 25-60 45-100

Infected Family $0-1$

The following calculation sets will help in understanding the above table:

International Travel $=\{$ Low, Medium, High $\}$

Low $=\{6,7,8,9,10\}$ returned months before Medium $=\{3,4,5,6,7\}$ returned months before High $=\{0,1,2,3,4\}$ returned months before

Age $=\{$ Low, Medium, High $\}$

Low $=\{0-30\}$ years of age

Medium $=\{25-60\}$ years of age

High $=\{45-100\}$ years of age

These inputs are dependent on the risk factor they impose, whereas cough, fever and breathing trouble is calculated on the degree and nature of illness. The infected family plays a major role in predicting the risk of infecting others and it is calculated in a simple yes or no binary fashion where 0 means no infected family member and 1 means at least one infected family member [18].

After these inputs have been entered in the fuzzy logic, the rules have been implemented and defuzzification process is started.

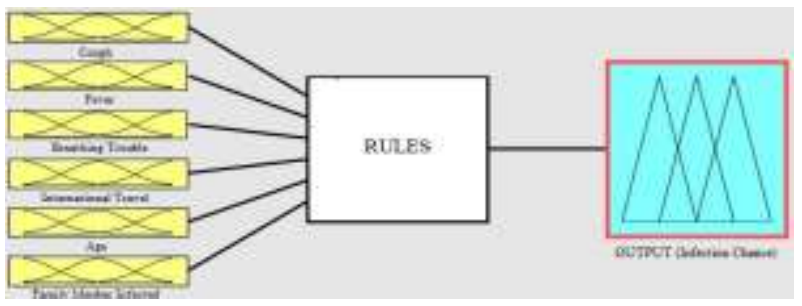

Fig. 2. The Fuzzy Logic Design for determining the chances of been affected by Corona Virus. 


\section{RESULT AND DISCUSSION}

The fuzzy logic has been designed after thorough study of research papers and information's released by World Health Organization (WHO). The rules have been set based on the number of people getting diagnosed by Corona Virus. According to scientists this virus is more dangerous for elderly people who are above the age of forty-five. Following statistics, the rules have been made and list of question are having been handed over to local people for survey. These surveys were inputted into the MATLAB fuzzy logic system and their infection percentage was predicted [19]. Survey questions were handed out to people with different backgrounds and having different age differences. All factors of transmission were taken into consideration for the evaluation and a proper prediction of chances of getting infected has been calculated in this paper.
A through survey had been conducted on eight states, two from Norther India, two from Southern India, two from Eastern India and two Western Indian states.

Table 2: States considered for Survey

\begin{tabular}{|c|c|c|}
\hline North & Jammu and Kashmir & Himachal Pradesh \\
\hline South & Kerala & Tamil Nadu \\
\hline East & West Bengal & Assam \\
\hline West & Gujrat & Maharashtra \\
\hline
\end{tabular}

The above table shows the states where the surveys were conducted. An averaged value of each parameter was considered and used as input in the fuzzy logic design from where the number of infected people in the next few days are predicted. The averaged values as obtained from the surveys are arranged in Descending values based on the percentage chance of getting infected:

Table 3: Averaged values used as input in Fuzzy Logic

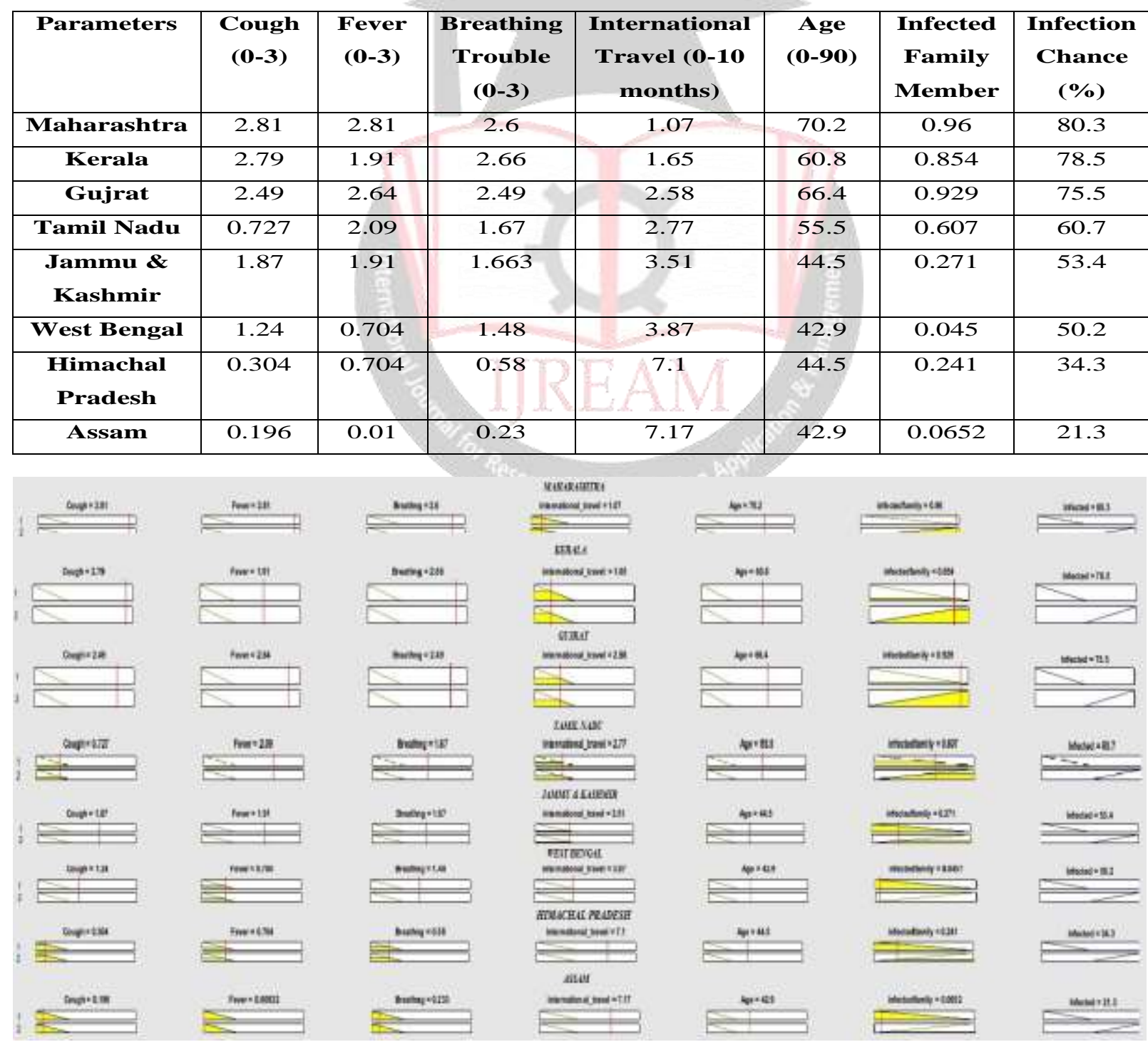

(a) 


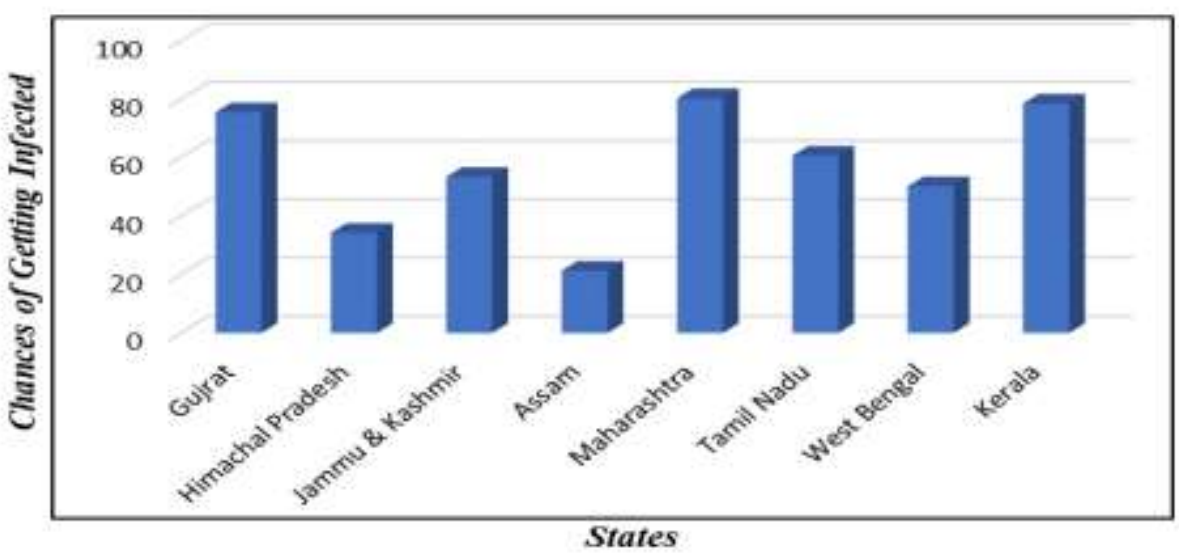

(b)

Fig. 3. (a) Fuzzy Logic interpretation and (b) State-wise Coronavirus infection prediction

The averaged value of each parameter has been put up in Fuzzy logic input. The values are shown in Table 1. Defuzzification of these inputs generated results, predicting the chances of a person to be infected by Coronavirus. Three membership functions were considered for generating results each of which ranged from High, Medium and Low. Figure (a) is the Fuzzy Logic Defuzzification which calculates the variable set as the input and generated an approximate prediction of a person to be affected by coronavirus. The predicted approximated result is represented on the extreme right side of the image. The states with highest probability are shown at the top which is followed by states with gradual decrease in percentage of chance of getting infected.

The above graphs as shown in figure "b" represent the column bar type representation of the percentage chance of getting infected by a person with Corona Virus. As per the data's revealed from survey it has been observed that people of Assam has least probability to get infected. Assam has till yet has a record of zero Corona virus case, whereas from the fuzzy logic interpretation it is observed that Assam has a probability of getting affected by corona virus by $20 \%$. It is observed that Maharashtra, Kerala and Gujrat have the highest chance of getting affected. This way we can utilize fuzzy logic method for predicting the spread of the disease. In a decreasing order of percentage chance of getting infected by COVID-19, the states which have been considered as our case study can be arranged as - Maharashtra, Kerala, Gujrat, Tamil Nadu, Jammu \& Kashmir, West Bengal, Himachal Pradesh and Assam.

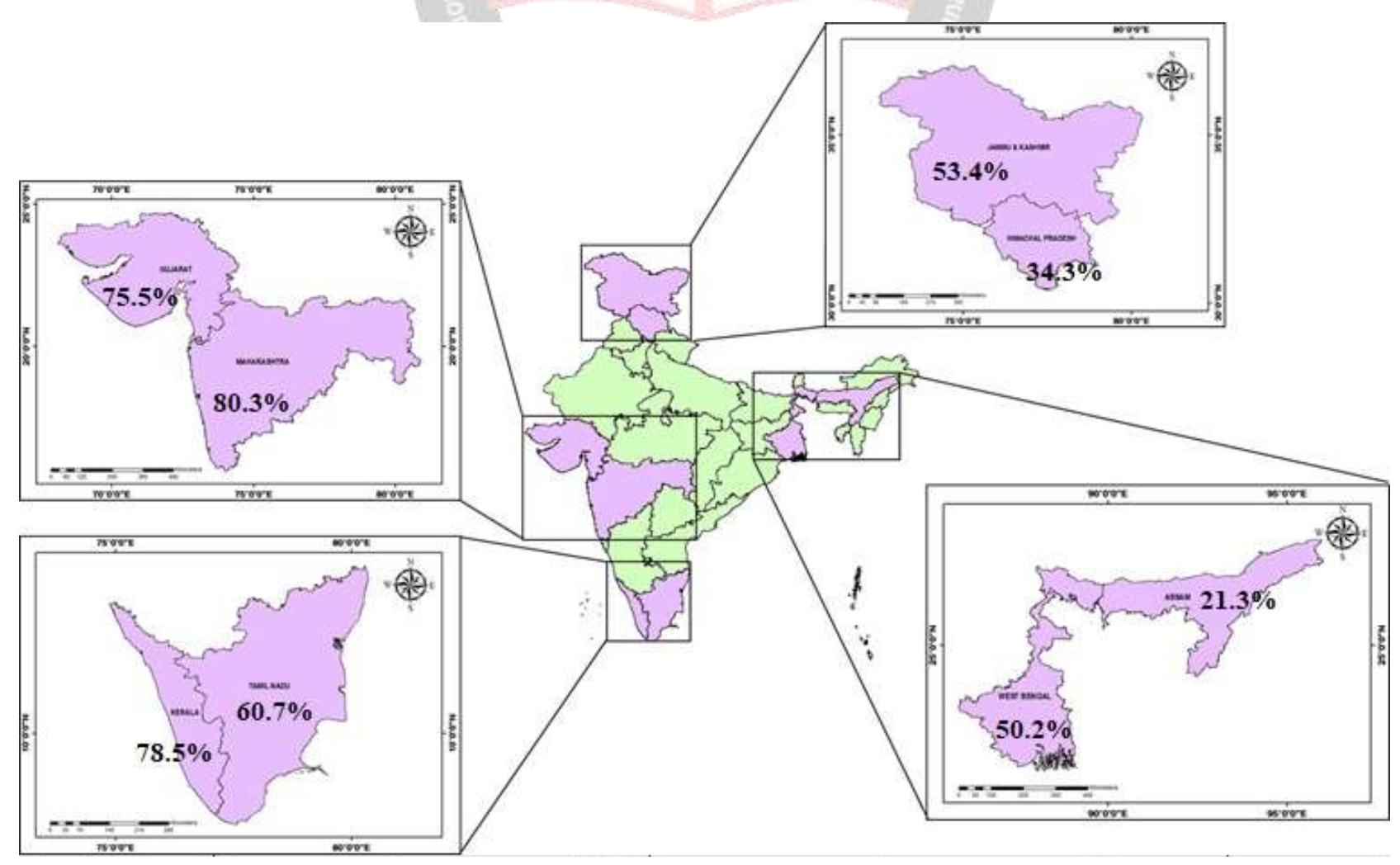

Fig. 1. State wise representation of Fuzzy Logic prediction 
The above figure (4) shows the state wise prediction of a person getting infected by Coronavirus as per fuzzy logic at a glance. The map has been created using ArcMap 10.3.1. Maharashtra being the center of main entertainment zone and business due to some cities like Mumbai attract a large number of tourists and business associates. Most of them have a comparatively higher international travel history making the residents of Mumbai highly probable of getting infected by coronavirus.

\section{CONCLUSION}

According to the survey conducted, it can be concluded that Maharashtra has the highest risk of getting infected followed by Kerala and Assam has the least probability of getting affected by Coronavirus. Maharashtra being predicted at the highest of all readings, $80.3 \%$ followed by Kerala at $78.5 \%$. Gujrat being the neighboring state of Maharashtra has lower possibility of been affected by Coronavirus. The steps which are taken by each state plays an important role in limiting the spread of Coronavirus. The use of Fuzzy Logic has proved to be useful for predicting how much an individual is prone to be affected by Coronavirus. This can be said since the predicted results from Fuzzy Logic defuzzification shows good relationship with original ongoing current scenario [https://www.covid19india.org/]. All the averaged-out data and values acquired from the surveys conducted, the output values were entered into the fuzzy logic inputs. Based on them fuzzy logic defuzzification generate results predicting the probability of a person getting infected by coronavirus. The fuzzy logic defuzzification shows high risk alert for Maharashtra and least by Assam. Assam being the closest to China among all the other states considered shows more probability of getting infected than the current situation in the world. It can be safely concluded that on the basis of the research work conducted, Western India has a more probably of getting infected by this pandemic disease. However, it should be kept in mind that proper precautions must be taken into consideration strictly to avoid more spreading of this pandemic disease.

\section{REFERENCES}

[1] Ferguson, Neil, Daniel Laydon, Gemma Nedjati Gilani, Natsuko Imai, Kylie Ainslie, Marc Baguelin, Sangeeta Bhatia et al. "Report 9: Impact of non-pharmaceutical interventions (NPIs) to reduce COVID19 mortality and healthcare demand." (2020).

[2] Singh, Harpreet, Madan M. Gupta, Thomas Meitzler, Zeng-Guang Hou, Kum Kum Garg, Ashu MG Solo, and Lotfi A. Zadeh. "Real-life applications of fuzzy logic." Advances in Fuzzy Systems 2013 (2013).

[3] Box, George EP. "Science and statistics." Journal of the American Statistical Association 71, no. 356 (1976): 791-799.

[4] https://www.who.int/health-topics/coronavirus\#tab=tab_1

[5] https://www.who.int/emergencies/diseases/novel-coronavirus-2019
[6] Singh, Rajesh, and R. Adhikari. "Age-structured impact of social distancing on the COVID-19 epidemic in India." arXiv preprint arXiv:2003.12055 (2020).

[7] "India's first coronavirus death is confirmed in Karnataka". Hindustan Times. 12 March 2020. Retrieved 27 March 2020.

[8] Keeling, Matt J., and Pejman Rohani. Modeling infectious diseases in humans and animals. Princeton University Press, 2011.

[9] Diekmann, Odo, J. A. P. Heesterbeek, and Michael G. Roberts. "The construction of next-generation matrices for compartmental epidemic models." Journal of the Royal Society Interface 7, no. 47 (2010): 873-885.

[10] https://www.worldometers.info/coronavirus/country/india/

[11] Lambovska, Maya. "A fuzzy model for team control and its application." Management and Production Engineering Review 9 (2018).

[12] Parker, Glenn M. Team players and teamwork: New strategies for developing successful collaboration. John Wiley \& Sons, 2011.

[13] https://www.aljazeera.com/news/2020/01/timeline-chinacoronavirus-spread-200126061554884.html

[14] https://www.health.harvard.edu/diseases-andconditions/coronavirus-resource-center

[15] https://www.cdc.gov/coronavirus/2019-ncov/prevent-gettingsick/how-covid-spreads.html

[16] Deptuła, Anna Małgorzata, and Katarzyna Rudnik. "Fuzzy approach using experts' psychological conditions to estimate the criteria importance for the assessment of innovative projects risk." Management and Production Engineering Review 9 (2018).

[17] Simiński, Krzysztof. "Merging of fuzzy models for neuro-fuzzy systems." Theoretical and Applied Informatics 23, no. 2 (2011): 107-126.

[18] Porębski, Sebastian, and Ewa Straszecka. "Membership functions for fuzzy focal elements." Archives of Control Sciences 26, no. 3 (2016): 395-427.

[19] Hosseini, Navid, and Mehran Gholinejad. "Investigating the slope stability based on uncertainty by using fuzzy possibility theory." Archives of Mining Sciences 59, no. 1 (2014): 179-188. 